\title{
Ultra-Wideband Time of Flight Based Localization System and Odometry Fusion for a Scanning 3 DoF Magnetic Field Autonomous Robot
}

\author{
Jose Lima ${ }^{1,3(\bowtie)}$ and Paulo Costa ${ }^{2,3}$ \\ 1 Polytechnic Institute of Bragança, Bragança, Portugal \\ jllima@ipb.pt \\ 2 Faculty of Engineering, University of Porto, Porto, Portugal \\ paco@fe.up.pt \\ 3 INESC-TEC, Centre for Robotics in Industry and Intelligent Systems, \\ Porto, Portugal
}

\begin{abstract}
Solving the robot localization problem is one of the most necessary requirements for autonomous robots. Several methodologies can be used to determine its location as accurately as possible. What makes this difficult is the existence of uncertainty in the sensing of the robot. The uncertain information needs to be combined in an optimal way. This paper stresses a Kalman filter to combine information from the odometry and Ultra Wide Band Time of Flight distance modules, which lacks the orientation. The proposed system validated in a real developed platform performs the fusion task which outputs position and orientation of the robot. It is used to localize the robot and make a 3 DoF scanning of magnetic field in a room. Other examples can be pointed out with the same localization techniques in service and industrial autonomous robots.
\end{abstract}

Keywords: Localization • Ultra Wideband Time of Flight • Autonomous mobile robot

\section{Introduction}

An industrial mobile robot requires the ability to self-localize in the environment without human intervention. This means answering the question "Where am I?" from the robot point of view.

Estimating the pose of the robot in a map has been capturing the attention of researchers and developers due to its complexity and the multitude of possible approaches.

Localization systems in industrial environments, are expensive and common to use solutions that rely on artificial landmarks, such as the classic magnetic tape following, line tracking or reflector based laser triangulation [1,2]. 
This method has a main disadvantage that requires the installation of dedicated reflectors for all the environment in the field of view of the robot laser scanner, that in some factories might become an complex and expensive solution.

Another localization method can be done resorting to radio frequency measuring the signal intensity (Receive Signal Strength Indicator, RSSI). Related problems such as distance errors and multipath effects can be identified in this approach. Instead, distances can be measured resorting to the transit time methodology (Time of Flight, ToF). This method measures the running time of light between a fixed tag module and receiver (Anchor). Ultra-wideband (UWB) modules from Decawave are used in this work. By this way, distances between different fixed tags and anchors can be determined. The distances allow to make the trilateration to estimate the tag position. Unfortunately, this system can only provide the position and not the orientation.

On the other hand, odometry provides the orientation but the cumulative error of localization is a well-known problem. To fulfill this problem, the information provided by odometry and by the UWB technology is further processed through a data fusion filter (Kalman filter).

This paper presents the data fusion of the UWB ToF distances with the odometry. The Kalman filter combines both information and provides as output the position and orientation data. This development allows to validate the concept of data fusion from odometry and UWB ToF distancies and it is implemented in a 3 DoF magnetic field sensor robot that scans over a desired path.

The paper is organized as follows: After a brief introduction, Sect. 2 presents the related work of localization having in mind the UWB technology. Then, Sect. 3 addresses the system architecture where the odometry and UWB are described and the UWB localization model is stressed. Section 4 presents the data fusion from odometry and UWB ToF data whereas Sect. 5 results of localization and magnetic field scanning are discussed. Finally last section concludes the paper and presents some future work.

\section{Related Work}

Once mobile robots localization is a complex, challenging and one of the most fundamental problems in the robotics field, there are several approaches in the community. Among the others, laser triangulation, matching algorithms, complex vision systems, odometry and radio frequency are methodologies used to find the position of a robot.

In the matching algorithms, position estimation is commonly fused with dead reckoning data, using for that purpose, probabilistic methods such as the Kalman Filter family and the Particle Filters. There are matching algorithms that require prior knowledge on the navigation area. This prior knowledge can be an environment map, natural landmarks or artificial beacons [3]. There are other types of matching algorithms, which compute the overlapping zone between consecutive observations to obtain the vehicle displacement. One possible matching algorithm to estimate the quantity of angular and linear displacement of a vehicle between two different and consecutive configurations is the Iterative Closest 
Point (ICP) $[5,6]$. The problem of this approach is the huge amount of data to be processed. The process of finding the correct correspondence between points (matching) is a difficult and time-consuming task.

Another common localization approach is to combine several solutions such as line following and laser triangulation [1,2]. Meanwhile, in the last decade localization based on natural marks has been increasing [7]. These natural marks are composed by a set of distances and angles to the detected objects (such as doors, walls, furniture, ... ) that can be acquired through an on-board laser range finder. This method has the main advantage of not requiring the installation of dedicated reflectors in the environment, which in some factories might not be a viable option. On the other hand, objects placed in different locations originate measuring errors.

Wireless distance measurement technologies are an increasingly important technology. High accuracy measurements are critical for in-building applications such as autonomous robotic navigation. In these cases, users are requiring ever greater levels of accuracy. Actually, sub-centimeter accuracy can be achieved [4].

The trilateration problem, the process of finding the center of the area of intersection of three spheres, demands a different treatment to other similar solutions, as for example, those based on laser. There are authors that characterize the UWB ranges combined with particle filters within a variety of environments and situations [12] and apply UWB using spatial models [8]. Ultrawideband (UWB) localization is a recent technology that promises to outperform many indoor localization methods currently available [9]. UWB has received some attention within the robotics community [17]. It is considered one of the most promising indoor positioning technologies currently available, especially due to its fine time resolution [8]. UWB time of flight has already captured the researchers attention that combines it with inertial sensors [10]. Unfortunately, the orientation cannot be determined with this approach.

Odometry has been used for several years in mobile robots. Knowing the rotation of each wheel and its parameters (such as diameter, distance, friction) it is possible to estimate the posture of the robot on the environment. This is one of the first methodology used to calculate the robot position. A common and basic localization method called dead reckoning (DR) is used to estimate the position by counting wheel rotations with the help of encoders.

However, there are unavoidable accumulated errors for DR based localization over long distances, in that it needs to utilize the previous position to estimate the next relative one and during which, the drift, the wheel slippage, the uneven floor and the uncertainty about the structure of the robot will together cause errors [15]. Due to this cumulative errors it is common to combine different localization methods. Several works can be pointed out using the odometry localization with different approches, such as image processing [13] or Wireless Sensor Network [14].

The presented work uses a combination of odometry and UWB ToF distance to compute the position and orientation of a robot. By this way, it is possible to localize (position and orientation) the robot and perform the magnetic 
field scanning in an autonomous way. There are some commercial products like automated scanners but they are remote operated vehicles and not autonomous (example Jireh Industries. As extended work, the localization system could be improved (another Kalman filter input) with the magnetic field acquired data, as shown in [11].

\section{System Architecture}

A wheeled mobile robot prototype of $28 \times 35 \mathrm{~cm}$ (Fig. 1) was developed having in mind the scanning of the magnetic field in a room based of the proposed positioning system.

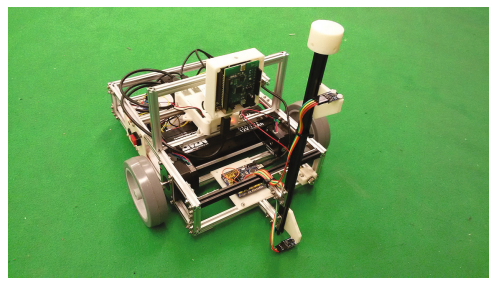

Fig. 1. Mobile robot prototype

It is composed by two drive wheels and a castor wheel. Two stepper motors drive the differential mobile robot, a typical configuration in mobile robots, are powered by Allegro MicroSystems - A4988 modules that handle the microstepping method and regulate the current. The maximum speed of the robot is $1 \mathrm{~m} / \mathrm{s}$ but the tests presented in Results section were achieved with $10 \%$ of speed. It is powered by onboard $12 \mathrm{~V}$ battery and a DC/DC step down converter allows to supply the electronic modules composed by a Raspberry 3 model and arduino microcontroller boards. The upper level is composed by a Raspberry microcomputer that runs raspbian operative system and is responsible for the Kalman filter processing, wi-fi communications and decision. The Arduino microcontroller

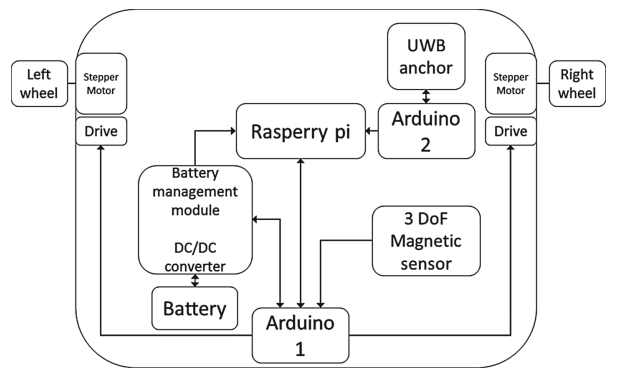

Fig. 2. Mobile platform architecture 
boards deal with the low level control of motors, voltages, current, power management, odometry and acquire the 3 axis magnetic field sensors (HMC5883L). A block diagram of the system is presented in Fig. 2.

\subsection{Ultra Wideband Time of Flight}

Time of flight describes methods that measure the time that an object, particle, electromagnetic or other wave take to travel a distance. It is a technology used, for example, in depth cameras that allows to measure the distance of an object to the camera based on the travel time of the speed of light. Using radio frequency, there are some approaches that estimates the distance measuring the signal strengths (Receive Signal Strength Indicator, RSSI). Results are not much satisfactory because signal reflections and multi-path effects introduces errors and noise in measure. The distance between two Ultra Wideband (UWB) devices can be measured precisely by measuring the time that it takes for a radio wave to pass between the two devices. It delivers much more precise distance measurement than signal-strength estimation. UWB signals maintain their integrity and structure even in the presence of noise and multi-path effects. It is a technology based on the IEEE 802.15.4-2011 standard, which can enable tagged objects to be located [16].

Decawave DW1000 is a single chip, UWB compliant, and Arduino compatible Wireless Transceiver based on Ultra Wideband techniques and provides a new approach to Real Time Location and Indoor Positioning Systems. This module, embedded in the Pozyx system is presented in Fig. 3. The presented hardware allows to measure the distance between modules process the (x,y) position.

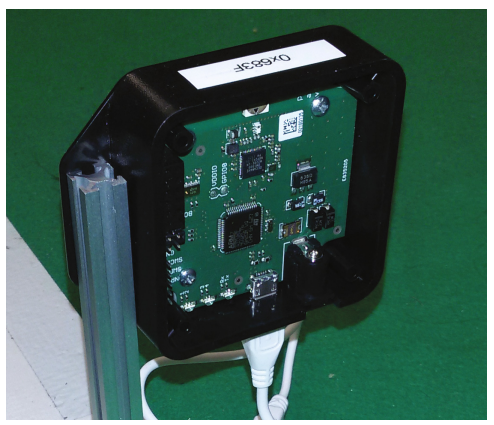

Fig. 3. UWB Pozyx tag

\subsection{UWB - ToF Distances Model}

The model of the Ultra wide band can be achieved through several measurements in the field where 512 samples of $(x, y)$ measures for 44 positions were acquired, 
having the tags placed in the bottom corners of a room of $7 \times 2.5 \mathrm{~m}$. A prefiltering process before Kalman filter (a median based gate, shown in Fig. 5) must be applied to reject the incoherent measures. In fact, the $x$ and $y$ errors remain the same but the co-variance is reduced with the proposed filter as presented in Table 1 where $R_{k}$ is the observation noise covariance that will be used in the Kalman filter. Figure 4 presents the error ellipses for both methods: at left without median filter gate and at right with it.

Table 1. Error and co-variance values with and without median based filter

\begin{tabular}{l|l|l}
\hline & Without median filter & With median filter \\
\hline error $\mathrm{x}$ & -0.079 & -0.074 \\
\hline error $\mathrm{y}$ & -0.013 & -0.013 \\
\hline co-variance & $R_{k}=\left[\begin{array}{cc}0.0433 & -0.0028 \\
-0.0028 & 0.0079\end{array}\right]$ & $R_{k}=\left[\begin{array}{ll}0.0015 & 0.0002 \\
0.0002 & 0.0005\end{array}\right]$ \\
\hline
\end{tabular}
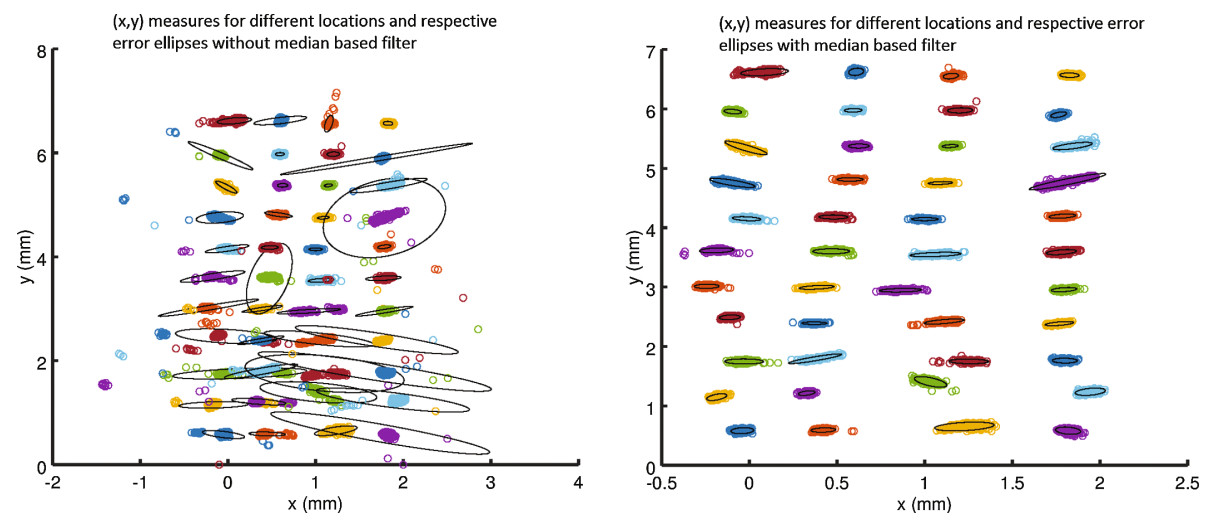

Fig. 4. (x,y) measures for different locations and respective error ellipses with and without median filter

\section{Localization Data Fusion}

This section presents the Kalman filter that receives the odometry and the UWB modules position information and outputs the position and orientation. The median based gate filters the position provided by the ultra wide band time of flight module (UWB-ToF), $x_{p}^{\prime}$ and $y_{p}^{\prime}$. The Kalman filter implements the sensor fusion task. It receives $x_{p}$ and $y_{p}$ (position) from the median based gate and $\omega_{L}$ 
and $\omega_{R}$ (left and right wheel speeds) from the odometry system. The output of the filter $\left(X_{k}\right.$ in Eq. 1 ) is composed by $x, y$ and $\theta$ that is the robot position and orientation as presented in Fig. 5. In this case, the height of all tags and anchor was the same.

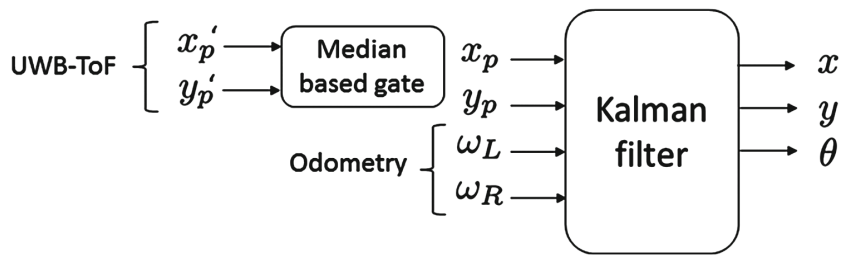

Fig. 5. Kalman filter inputs/outputs.

The first filtering approach, the median filter presented in previous section, is applied. The filtered values ( $\mathrm{x}$ and $\mathrm{y}$ values to set the center of the gate that eliminates unlikely measures) are the input for the Kalman filter.

$$
\begin{gathered}
X_{k}=\left[\begin{array}{l}
x \\
y \\
\theta
\end{array}\right] \\
\dot{X}=f(X, u)
\end{gathered}
$$

where

$$
u=\left[\begin{array}{c}
v \\
\omega
\end{array}\right]
$$

The observation estimate, $Z$ :

$$
Z=h(X)
$$

From odometry (Fig. 6), $v_{L}=\omega_{L} \cdot r$ and $v_{R}=\omega_{R} \cdot r$ where $r$ is the wheel radius.

The linear velocity $(v)$ and angular velocity $(\omega)$ can be calculated as presented in Eq. 5 where $d$ is the distance between wheels that equals $0.29 \mathrm{~m}$.

$$
v=\frac{v_{L}+v_{R}}{2} \quad \omega=\frac{v_{R}-v_{L}}{d}
$$

Equation $2(\dot{X})$ can now be calculated as:

$$
\left[\begin{array}{c}
\dot{x} \\
\dot{y} \\
\dot{\theta}
\end{array}\right]=\left[\begin{array}{c}
v \cdot \cos (\theta) \\
v \cdot \sin (\theta) \\
\omega
\end{array}\right]
$$




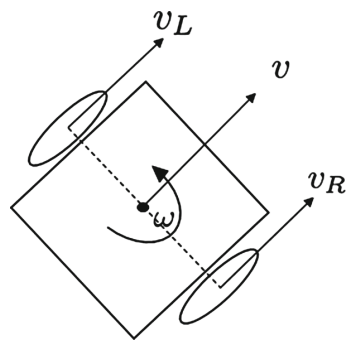

Fig. 6. Robot velocity vectors.

$F_{k}$ is the state transition model which is applied to the previous state $X_{k-1}$, expressed in Eq. 7 where $\Delta t$ is the time step for the acquisition.

$$
F_{k}=\left[\begin{array}{ccc}
1 & 0 & -\Delta t \cdot v \cdot \sin (\theta) \\
0 & 1 & \Delta t \cdot v \cdot \cos (\theta) \\
0 & 0 & 1
\end{array}\right]
$$

The Jacobian of $h$ function is:

$$
H_{k}=\left[\begin{array}{lll}
1 & 0 & 0 \\
0 & 1 & 0
\end{array}\right]
$$

The measurement from UWB-ToF is $Z_{k}$ :

$$
Z_{k}=\left[\begin{array}{l}
x_{p} \\
y_{p}
\end{array}\right]
$$

Predict. This subsection addresses the predict of state.

The predict covariance estimate, $P_{k}^{-}$(until $k$ instant).

$$
P_{k}^{-}=F_{k-1} P_{k-1} F_{k-1}^{\prime}+Q_{k-1}
$$

where $F_{k}$ is the state transition model which is applied to the previous state $x_{k-1}$ and $Q_{k-1}$ is the process noise covariance. The $Q_{k-1}$ matrix can be written as Eq. 11.

$$
Q_{k-1}=\left[\begin{array}{ccc}
\operatorname{cov}\left(v_{x}, v_{x}\right) & \operatorname{cov}\left(v_{x}, v_{y}\right) & \operatorname{cov}\left(v_{x}, \omega\right) \\
\operatorname{cov}\left(v_{x}, v_{y}\right) & \operatorname{cov}\left(v_{y}, v_{y}\right) & \operatorname{cov}\left(v_{y}, \omega\right) \\
\operatorname{cov}\left(v_{x}, \omega\right) & \operatorname{cov}\left(v_{y}, \omega\right) & \operatorname{cov}(\omega, \omega)
\end{array}\right]
$$

Instead of working with $v_{x}$ and $v_{y}$, it is possible to perform a rotation $R o t$ (Eq. 12) to work with $v$ and $v_{n}$ (where $v$ is the linear velocity whereas $v_{n}$ is the normal velocity) we can assume $v_{n}=0$ and $\operatorname{cov}\left(v_{n}, v_{n}\right)=\sigma_{n}^{2}$.

$$
\left[\begin{array}{l}
v_{x} \\
v_{y} \\
\omega
\end{array}\right]=\left[\begin{array}{ccc}
\cos (\theta) & -\sin (\theta) & 0 \\
\sin (\theta) & \cos (\theta) & 0 \\
0 & 0 & 1
\end{array}\right] \cdot\left[\begin{array}{c}
v \\
v_{n} \\
\omega
\end{array}\right]
$$


Working with $v$ and $v_{n}, Q_{k-1}=\operatorname{Rot} \cdot Q_{k-1}^{\prime} \cdot \operatorname{Rot}^{T}$, where $Q_{k-1}^{\prime}$ is presented in Eq. 13, it is possible to reach the ratio between $\operatorname{cov}(v, v)$ and $\operatorname{cov}(\omega, \omega)$ presented in Eq. 16, assuming that $v_{L}$ and $v_{R}$ errors follows a normal distribution $(\mathrm{N})$ centred in zero with a standard deviation of $\sigma_{o}$. Equations 14 and 15 present the distribution of $v$ and $\omega$.

$$
\begin{gathered}
Q_{k-1}^{\prime}=\left[\begin{array}{ccc}
\operatorname{cov}(v, v) & 0 & 0 \\
0 & \operatorname{cov}\left(v_{n}, v_{n}\right) & 0 \\
0 & 0 & \operatorname{cov}(\omega, \omega)
\end{array}\right] \\
v \rightarrow 2 \cdot \frac{1}{4} \cdot N\left(0, \sigma_{o}^{2}\right) \\
\omega \rightarrow 2 \cdot \frac{1}{d^{2}} \cdot N\left(0, \sigma_{o}^{2}\right) \\
\operatorname{cov}(v, v)=\frac{4}{d^{2}} \operatorname{cov}(\omega, \omega)
\end{gathered}
$$

This methodology allows to tune the Kalman filter by two constants $\left(\sigma_{o}, \sigma_{n}\right)$ that can be found by performing a few experiences.

The prediction state estimate, $X_{k}^{-}$(until $k$ instant)

$$
X_{k}^{-}=f^{*}\left(X_{k-1}, u_{k-1}\right)
$$

Update. This subsection addresses the Update process.

The measurement residual:

$$
\tilde{Y}_{k}=Z_{k}-Z
$$

The innovation covariance:

$$
S_{k}=H_{k} P_{k}^{-} H_{k}^{\prime}+R_{k}
$$

where $R_{k}$ is the observation noise covariance, that can be calculated based on the covariance average of measures points from previous subsection as presented in Table 1.

The Kalman gain, $K_{k}$ :

$$
K_{k}=P_{k}^{-} H_{k}^{\prime} S_{k}^{-1}
$$

The updated state estimate, $X_{k}$ :

$$
X_{k}=X_{k}^{-}+K_{k} \tilde{Y}_{k}
$$

The updated covariance estimate, $P_{k}$ can be described:

$$
P_{k}=\left(I-K_{k} H_{k}\right) P_{k}^{-}
$$




\section{Results}

The localization system under validation was tested in the developed robot on a corridor of $7 \mathrm{~m} \times 2.5 \mathrm{~m}(\mathrm{x}, \mathrm{y})$. The output state $X_{k}$ of the Kalman filter allows to control the robot to follow the desired path presented in Fig. 7 (the controller will be addressed in a future work). The tags were placed in the corners, as presented in Fig. 7 by blue circles and the scanning was performed according to the presented path. Start position is $(0,0.5)$ and the end position is $(2,0.5)$.

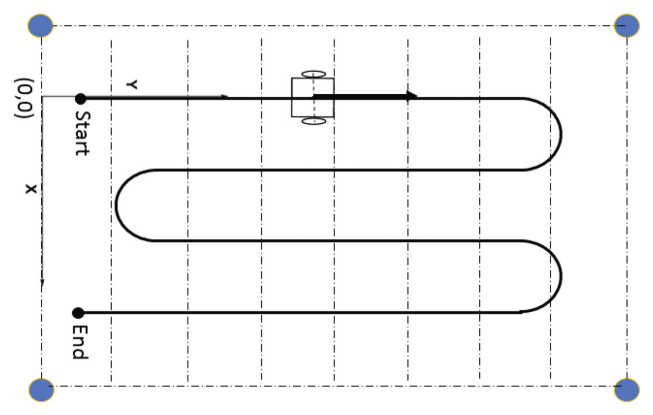

Fig. 7. Scanning path

Obviously, this is only for localization demonstration purpose. Otherwise, a more detailed scan could be performed. Euclidean Mean absolute error associated with the proposed methodology is about $6 \mathrm{~cm}$. A ground truth system will be applied to validate the presented approach as future work. The scan with the 3 DoF magnetic field sensor allows to measure the magnetic field value in $x, y$ and $z$ directions. Figure 8 presents the direction of the $x$ and $y$ vectors whereas Fig. 9 presents the $x, y$ and $z$ vectors magnitude.

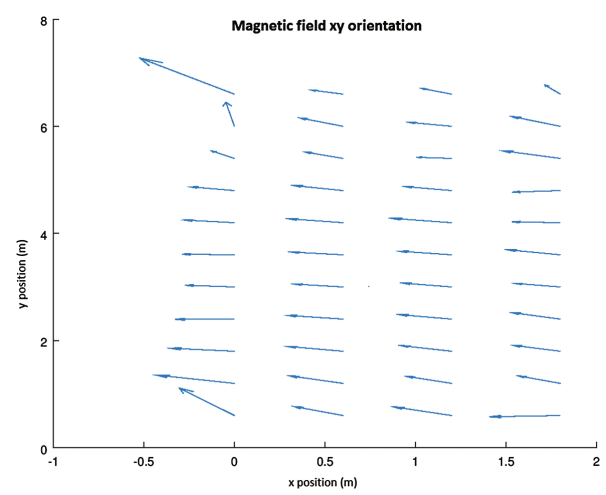

Fig. 8. Magnetic field orientation vector 
The disturbances in the magnetic field orientation and magnitude are explained by metallic underground boxes.

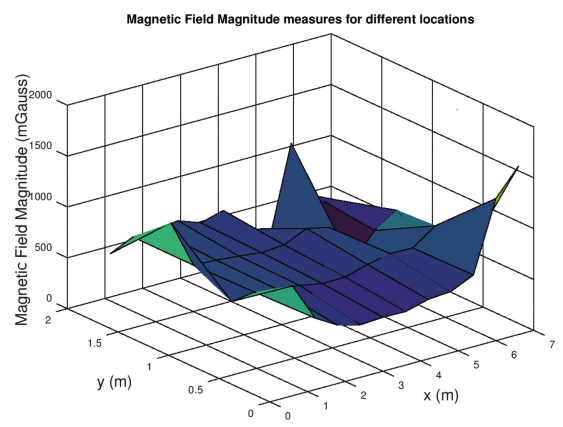

Fig. 9. Magnetic field magnitude plot

\section{Conclusion and Future Work}

The presented paper proposes a robot localization system based on odometry and ultra wideband time-of-flight. This sensor fusion allows to estimate the orientation of the robot that UWB ToF lacks. The data fusion is processed by a Kalman filter and the implementation of a pre filtering system composed by a median based gate allows to reduce the covariance. Thus, localization error is reduced. As implementation, a 3 DoF magnetic field scan is done in a floor of a corridor that finds the underground hidden metallic objects.

As future work, Kalman filter inputs can be added such as the previous known scanned magnetic field, accelerometer/gyroscope and visual localization to improve the localization of the robot. Also, a ground truth system will be applied to validate the presented approach.

Acknowledgment. Project "TEC4Growth - Pervasive Intelligence, Enhancers and Proofs of Concept with Industrial Impact/NORTE-01-0145-FEDER-000020" is financed by the North Portugal Regional Operational. Programme (NORTE 2020), under the PORTUGAL 2020 Partnership Agreement, and through the European Regional Development Fund (ERDF). This work is also financed by the ERDF European Regional Development Fund through the Operational Programme for Competitiveness and Internationalisation - COMPETE 2020 Programme, and by National Funds through the FCT Fundaçao para a Ciência e a Tecnologia (Portuguese Foundation for Science and Technology) within project POCI-01-0145-FEDER-006961. 


\section{References}

1. Schulze, L., A.: Wullner, A.: The approach of automated guided vehicle systems. In: 2006 IEEE International Conference on Service Operations and Logistics, and Informatics, pp. 522-527 (2006)

2. Schulze, L., Behling, S., Buhrs, S.: Automated guided vehicle systems: a driver for increased business performance. In Proceedings of International Multiconference of Engineers and Computer Scientists, pp. 19-21 (2008)

3. Lauer, M., Lange, S., Riedmiller, M.: Calculating the perfect match: an efficient and accurate approach for robot self-localization. In: RoboCup 2005: Robot Soccer World Cup IX, pp. 142-153 (2006)

4. Petroff, A.M.: Ultra wideband two-way time-of-flight distance measurement provides sub-centimeter range measurement accuracy. In: Radio Science Meeting (Joint with AP-S Symposium) (2015)

5. Besl, P.J., McKay, H.D.: A method for registration of 3-D shapes. IEEE Trans. Pattern Anal. Mach. Intell. 14(2), 239-256 (1992)

6. Censi, A.: An ICP variant using a point-to-line metric. In: 2008 IEEE International Conference on Robotics and Automation, pp. 19-25 (2008)

7. Tomatis, N.: BlueBotics: navigation for the clever robot [entrepreneur]. IEEE Robot. Autom. Mag. 18(2), 14-16 (2011)

8. Prorok, A., Martinoli, A.: Accurate indoor localization with ultra-wideband using spatial models and collaboration. Int. J. Robot. Res. 33(4), 547-568 (2014)

9. Prorok, A., Martinoli, A.: Accurate localization with ultra-wideband: tessellated spatial models and collaboration. In: 13th International Symposium on Experimental Robotics (2013)

10. Kok, M., Hol, J.D., Schön, T.B.: Indoor positioning using ultrawideband and inertial measurements. IEEE Trans. Veh. Technol. 64(4), 1293-1303 (2015)

11. Rahok, S.A., Shikanai, Y., Ozaki, K.: Trajectory tracking using environmental magnetic field for outdoor autonomous mobile robots. In: IEEE/RSJ International Conference on Intelligent Robots and Systems, Taiwan (2010)

12. Gonzalez, J., Blanco, J.L., Galindo, C., Ortiz-de-Galisteo, A., Fernandez-Madrigal, J.A., Moreno, F.A., Martinez, J.L.: Mobile robot localization based on ultra-wideband ranging: a particle filter approach. Robot. Auton. Syst. 57(5), 496-507 (2009)

13. Pizarro, D., Mazo, M., Santiso, E., Marron, M., Jimenez, D., Cobreces, S., Losada, C.: Localization of mobile robots using odometry and an external vision sensor. Sensors 10, 3655-3680 (2010)

14. Fu, G., Zhang, J., Chen, W., Peng, F., Yang, P., Chen, C.: Precise localization of mobile robots via odometry and wireless sensor network. Int. J. Adv. Rob. Syst. 10, 203-213 (2013)

15. Borenstein, J., Feng, L.: Measurement and correction of systematic odometry errors in mobile robots. IEEE Trans. Robot. Autom. 12(6), 869-880 (1996)

16. Decawave website: UWB transceiver (2017). http://www.decawave.com/

17. Hollinger, G.A., Djugash, J., Singh, S.: Target tracking without line of sight using range from radio. Auton. Rob. 32(1), 1-14 (2012) 